\title{
Application Article \\ Joint Optimization of Microstrip Patch Antennas Using Particle Swarm Optimization for UWB Systems
}

\author{
Muhammad Zubair and Muhammad Moinuddin \\ Faculty of Engineering, IQRA University, Karachi 75500, Pakistan \\ Correspondence should be addressed to Muhammad Zubair; zubair@iqra.edu.pk
}

Received 31 December 2012; Revised 26 April 2013; Accepted 27 April 2013

Academic Editor: Hon Tat Hui

Copyright ( 2013 M. Zubair and M. Moinuddin. This is an open access article distributed under the Creative Commons Attribution License, which permits unrestricted use, distribution, and reproduction in any medium, provided the original work is properly cited.

\begin{abstract}
Ultra wideband (UWB) systems are the most appropriate for high data rate wireless transmission with low power consumption. However, the antenna design for UWB has been a challenging task. Moreover, it is always desirable to have more freedom by designing different shape antennas with identical characteristics so that they can be used in either transmitter or receiver depending on other physical constraints such as area. To tackle these issues, in this paper, we have investigated a joint optimization of three different shape-printed monopole antennas, namely, printed square monopole antenna, printed circular monopole antenna and printed hexagonal monopole antenna, for UWB applications. More specifically, we have obtained the optimized geometrical parameters of these antennas by minimizing the mean-square-error for desired lower band edge frequency, quality factor, and bandwidth. The objective of joint optimization is to have identical frequency characteristics for the aforementioned three types of PMA which will give a freedom to interchangeably use them at either side, transmitting or receiving. Moreover, we employ particle swarm optimization (PSO) algorithm for our problem as it is well known in the literature that PSO performs well in electromagnetic and antenna applications. Simulation results are presented to show the performance of the proposed design.
\end{abstract}

\section{Introduction}

Ultra wideband systems are most widely used wireless systems nowadays for high data rate applications. It consumes low power against extremely wide spectrum of radio frequency [1]. The UWB system was originally designed to be used in radar communication; however, it is now equally employed in various consumer products within short distances.

The Federal Communications Commission (FCC) has allocated a bandwidth of $7.5 \mathrm{GHz}$ (from $3.1 \mathrm{GHz}$ to $10.6 \mathrm{GHz}$ ) for UWB in order to minimize its interference with other communication systems [2].

Microstrip patch antennas are widely used for wide bandwidth and omni-directional applications. One of its various types is printed monopole antenna, which is available in many geometrical shapes [3-6]. These different shapes have different performance and gains at different central frequency.
The design of suitable antenna for UWB application is a major challenge. In order to operate various types of antenna at the same frequency, their optimum geometrical dimensions must be jointly evaluated. This is treated as a complex optimizing problem. It is extremely difficult to find optimum solution of a complex problem by employing traditional and conventional approaches. Various heuristic approaches are available to handle such types of complex problems. Among many approaches, particle swarm optimization is one of the fastest heuristic approaches for finding joint optimum solution of a complex problem.

In the literature, it is a very common practice to optimize either radiation properties (such as return loss bandwidth, return loss magnitude) or resonant frequency to calculate the optimized geometrical parameters of the antenna. There are many published works which employ the similar strategy for the optimization of antenna. They used the optimization to get geometrical parameters in order to meet certain desired frequency of operation. 
For example, gravitational search algorithm (GSA) has been applied for calculating the optimized length and width of rectangular patch antenna for a desired frequency [7]. In [8], resonant frequency of circular microstrip antenna, with and without air gaps, is optimized to calculate the different geometry parameters of the antenna, and in [9], particle swarm optimization technique is applied in conjunction with the developed standard equation to accurately calculate the resonant frequency and feed point of rectangular microstrip patch antenna. However, the uniqueness and the main contribution of this work are mentioned in following Section 1.1.

In our work, we are optimizing the resonant frequency to obtain the geometrical parameters of the three different printed monopole antennas for UWB applications. This paper focuses on jointly optimizing three different shaped PMAs, namely, square, circular, and hexagonal, for the effective use at the same frequency in the range of UWB spectrum. PSO algorithm is used as the optimizing technique. The "common" lower band edge frequency $\left(f_{L}\right)$, bandwidth $(B W)$, quality factor $(Q)$, and the joint cost function (mean square error) for all three antennas are shown in the end.

1.1. Our Contribution and Contrast to Existing Work. The main contributions and some contrast to existing works are outlined as follows.

(1) Unlike the most existing works which use individual optimization, our work employs joint optimization strategy to design different printed monopole antennas for UWB applications.

(2) We employ the PSO technique for our problem which has never been applied for such scenario.

(3) Few recent works have employed PSO but they did individual optimization in the same scenario of multiband or general wideband, not for UWB applications [10-12].

(4) Advantage of using joint optimization is that it develops different shape antennas with identical desired characteristics which give us the flexibility to use them according to our requirements and physical constraints. For example, one antenna can have identical frequency characteristics but different physical area. This can be observed in Tables 1, 2, and 3 .

(5) Finally, our work provides a unified framework which can be used in the future for designing any shape antenna in any range of applications.

1.2. Organization of the Paper. The above section briefly describes the aim and objective of this work. It includes the importance of the UWB system and its range. Moreover, it refers a few of the existing works on the optimization of patch antenna and describes the uniqueness of this work from other existing approaches. Section 2 explains different shapes of microstrip patch antenna. It describes the detailed geometrical parameters of three targeted antennas with their relevant equations. The next section explains the PSO algorithm in detail with their essential steps. Finally, the last section develops a joint cost function for the three targeted antennas on the basis of PSO approach. It also gives the optimized results in graphical and tabular form to show the successful implementation of the optimization approach.

\section{Parameters of Printed Monopole Antenna}

As discussed; the PMA are available in different regular geometrical shapes such as printed square monopole antenna (PSMA), printed rectangular monopole antenna (PRMA), printed hexagonal monopole antenna (PHMA), printed triangular monopole antenna (PTMA), printed circular monopole antenna (PCMA), and printed elliptical monopole antenna (PEMA) [13]. All these are shown in Figure 1.

The objective is to jointly optimize three basic shapes among the list of the six shapes of PMA (see Figure 1). The three targeted antennas are PSMA, PCMA, and PHMA. By using joint cost function (of error) through particle swarm optimization, suitable geometrical parameters of all three antennas are found. The calculated parameters are of such "optimum" values which enable these different shaped PMAs to operate equally well at the same frequency.

Instead of resonance frequency, the most significant parameters for UWB system are the lower band edge frequency $\left(f_{L}\right)$ and the bandwidth (BW). Printed monopole antenna is the best choice for UWB application. The lower band edge frequency of all types of PMAs with different feed configurations can be estimated using cylindrical monopole of large effective diameter.

A generalized modified relation for lower band frequency of all types of PMA can be written as [5]

$$
f_{L}=\frac{7.2}{[(L+r+p) \times k]} \mathrm{GHz},
$$

where $L$ is the height of planar monopole antenna which is taken as equivalent cylindrical monopole. The effective radius of equivalent cylindrical monopole is denoted by $r$. The symbol $p$ is the length of $50 \Omega$ feed line from the ground surface to the printed patch. Whereas $k$ is the empirical constant of the substrate. The commonly used substrate for printed patch is FR4 with dielectric constant $\epsilon_{r}=4.4$ and height $h=0.159 \mathrm{~cm}$. The value of empirical constant $k$ for this substrate is 1.15. All the variables $L, r$, and $p$ are measured in $\mathrm{cm}$ [13].

As discussed, PSO is applied to jointly optimize three different patch antennas PSMA, PCMA, and PHMA. Equation (1) is the generic equation for lower band edge frequency of all types of PMA. The equation can be rewritten for each specific type of antenna by considering their specific geometrical shapes and equating their dimensions with the variables given in (1). 
TABLE 1: Optimized values for PSMA at the lower frequency of 3.1, 4.8, 7.4, 8.5, and 10.2 GHz.

\begin{tabular}{|c|c|c|c|c|c|}
\hline \multirow{2}{*}{ Sr. No. } & \multirow{2}{*}{ Observed parameters } & \multirow{2}{*}{ Desired } & \multirow{2}{*}{ Calculated } & \multicolumn{2}{|c|}{ Geometric dimensions } \\
\hline & & & & $L$ & $p$ \\
\hline \multirow{3}{*}{1} & Frequency & 3.10 & 3.099522903 & \multirow{3}{*}{1.159845629} & \multirow{3}{*}{0.682043476} \\
\hline & Bandwidth & 7.50 & 7.510477097 & & \\
\hline & Quality factor & 0.76 & 0.761958146 & & \\
\hline \multirow{3}{*}{2} & Frequency & 4.80 & 4.800982534 & \multirow{3}{*}{0.597733983} & \multirow{3}{*}{0.605804566} \\
\hline & Bandwidth & 5.80 & 5.799017466 & & \\
\hline & Quality factor & 1.23 & 1.236991469 & & \\
\hline \multirow{3}{*}{3} & Frequency & 7.40 & 7.402219779 & \multirow{3}{*}{0.377047302} & \multirow{3}{*}{0.408068462} \\
\hline & Bandwidth & 3.20 & 3.198780221 & & \\
\hline & Quality factor & 2.77 & 2.776367057 & & \\
\hline \multirow{3}{*}{4} & Frequency & 8.50 & 8.530486913 & \multirow{3}{*}{0.353992207} & \multirow{3}{*}{0.321891931} \\
\hline & Bandwidth & 2.10 & 2.089513087 & & \\
\hline & Quality factor & 4.52 & 4.645127724 & & \\
\hline \multirow{3}{*}{5} & Frequency & 10.20 & 10.199922399 & \multirow{3}{*}{0.398869314} & \multirow{3}{*}{0.151464075} \\
\hline & Bandwidth & 0.40 & 0.400077601 & & \\
\hline & Quality factor & 26.00 & 25.990050825 & & \\
\hline
\end{tabular}

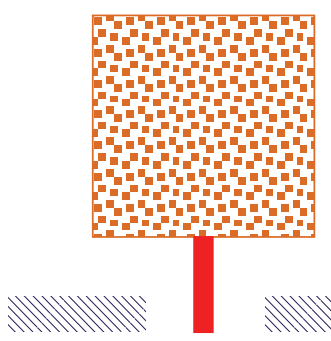

(a)

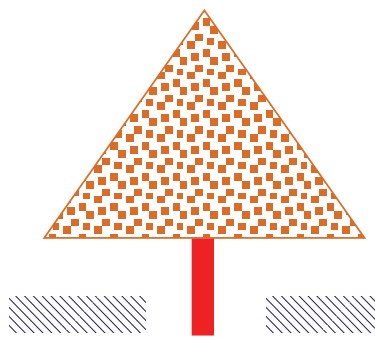

(d)

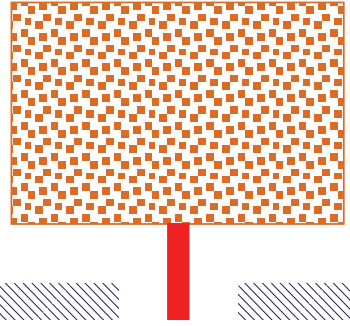

(b)

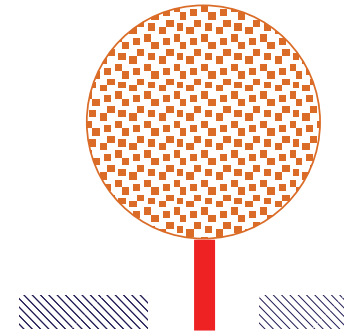

(e)

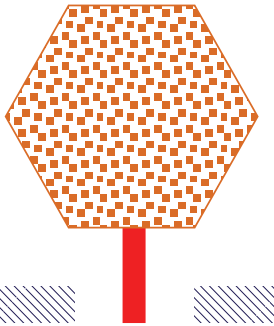

(c)

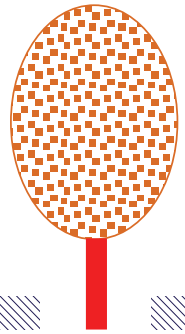

(f)

Figure 1: Printed (a) square, (b) rectangular, (c) hexagonal, (d) triangular, (e) circular, and (f) elliptical monopole antenna.

2.1. Printed Square Monopole Antenna. Since (1) is the generic equation for all PMAs, the variables $L$ and $r$ for PSMA are given as [13]

$$
\begin{gathered}
L=W(\text { width }) \mathrm{cm}, \\
r=\frac{L}{2 \pi} \mathrm{cm} .
\end{gathered}
$$

In the light of above relations, (1) for PSMA becomes

$$
f_{L}=\frac{14.4 \pi}{[L(2 \pi+1)+2 \pi p] k} \mathrm{GHz}
$$

where $L$ is the length of square monopole antenna as shown in Figure 2 and $p$ and $k$ are the same as generally described for (1).

2.2. Printed Circular Monopole Antenna. The variables $L$ and $r$ for PCMA are given as [13]

$$
\begin{aligned}
L & =2 A \mathrm{~cm}, \\
r & =\frac{A}{4} \mathrm{~cm} .
\end{aligned}
$$


TABLE 2: Optimized values for PCMA at the lower frequency of 3.1, 4.8, 7.4, 8.5, and 10.2 GHz.

\begin{tabular}{|c|c|c|c|c|c|}
\hline \multirow{2}{*}{ Sr. No. } & \multirow{2}{*}{ Observed parameters } & \multirow{2}{*}{ Desired } & \multirow{2}{*}{ Calculated } & \multicolumn{2}{|c|}{ Geometric dimensions } \\
\hline & & & & $A$ & $p$ \\
\hline \multirow{3}{*}{1} & Frequency & 3.10 & 3.099129460 & \multirow{3}{*}{1.113653073} & \multirow{3}{*}{0.830078783} \\
\hline & Bandwidth & 7.50 & 7.512870540 & & \\
\hline & Quality factor & 0.76 & 0.773128898 & & \\
\hline \multirow{3}{*}{2} & Frequency & 4.80 & 4.798244915 & \multirow{3}{*}{0.894574369} & \multirow{3}{*}{0.631623825} \\
\hline & Bandwidth & 5.80 & 5.871755085 & & \\
\hline & Quality factor & 1.23 & 1.266451419 & & \\
\hline \multirow{3}{*}{3} & Frequency & 7.40 & 7.379833139 & \multirow{3}{*}{2.827968575} & \multirow{3}{*}{0.309353270} \\
\hline & Bandwidth & 3.20 & 3.209668615 & & \\
\hline & Quality factor & 2.77 & 2.652668673 & & \\
\hline \multirow{3}{*}{4} & Frequency & 8.50 & 8.543197570 & \multirow{3}{*}{0.542374955} & \multirow{3}{*}{0.313923482} \\
\hline & Bandwidth & 2.10 & 2.125680243 & & \\
\hline & Quality factor & 4.52 & 4.599305466 & & \\
\hline \multirow{3}{*}{5} & Frequency & 10.20 & 10.189734201 & \multirow{3}{*}{0.237655476} & \multirow{3}{*}{0.119453729} \\
\hline & Bandwidth & 0.40 & 0.401764830 & & \\
\hline & Quality factor & 26.00 & 26.983456210 & & \\
\hline
\end{tabular}

TABLE 3: Optimized values for PHMA at the lower frequency of 3.1, 4.8, 7.4, 8.5, and 10.2 GHz.

\begin{tabular}{|c|c|c|c|c|c|}
\hline \multirow{2}{*}{ Sr. No. } & \multirow{2}{*}{ Observed parameters } & \multirow{2}{*}{ Desired } & \multirow{2}{*}{ Calculated } & \multicolumn{2}{|c|}{ Geometric dimensions } \\
\hline & & & & $H$ & $p$ \\
\hline \multirow{3}{*}{1} & Frequency & 3.10 & 3.101416659 & \multirow{3}{*}{0.511759365} & \multirow{3}{*}{0.931935721} \\
\hline & Bandwidth & 7.50 & 7.513583341 & & \\
\hline & Quality factor & 0.76 & 0.793112080 & & \\
\hline \multirow{3}{*}{2} & Frequency & 4.80 & 4.812380337 & \multirow{3}{*}{0.267555702} & \multirow{3}{*}{0.756623112} \\
\hline & Bandwidth & 5.80 & 5.793619663 & & \\
\hline & Quality factor & 1.23 & 1.256119482 & & \\
\hline \multirow{3}{*}{3} & Frequency & 7.40 & 7.429916694 & \multirow{3}{*}{0.232594737} & \multirow{3}{*}{0.362174730} \\
\hline & Bandwidth & 3.20 & 3.170083306 & & \\
\hline & Quality factor & 2.77 & 2.961862883 & & \\
\hline \multirow{3}{*}{4} & Frequency & 8.50 & 8.500091680 & \multirow{3}{*}{0.205209433} & \multirow{3}{*}{0.32340776} \\
\hline & Bandwidth & 2.10 & 2.110208320 & & \\
\hline & Quality factor & 4.52 & 4.679466376 & & \\
\hline \multirow{3}{*}{5} & Frequency & 10.20 & 10.211035670 & \multirow{3}{*}{0.176532980} & \multirow{3}{*}{0.276123401} \\
\hline & Bandwidth & 0.40 & 0.399963210 & & \\
\hline & Quality factor & 26.00 & 26.912348760 & & \\
\hline
\end{tabular}

Using the above values, (1) for PCMA becomes

$$
f_{L}=\frac{28.8}{[9 A+4 p] k} \mathrm{GHz},
$$

where $A$ is the radius of circular monopole antenna as shown in Figure 2 and $p$ and $k$ are the same as generally described for (1).

2.3. Printed Hexagonal Monopole Antenna. The variables $L$ and $r$ for PHMA are given as [13]

$$
\begin{aligned}
L & =\sqrt{3} H \mathrm{~cm}, \\
r & =\frac{3 H}{4 \pi} \mathrm{cm} .
\end{aligned}
$$

Using the above relations, (1) for PHMA becomes

$$
f_{L}=\frac{28.8 \pi}{[24.76559237 H+4 \pi p] k} \mathrm{GHz},
$$

where $H$ is the length of the sides of hexagonal monopole antenna as shown in Figure 2 and $p$ and $k$ are the same as generally described for (1).

All the above equations (3), (5), and (7) will be used to evaluate geometrical parameters of the above targeted antennas at the same lower band edge frequency.

The quality factor $(Q)$ of ultra wideband antenna sets the limits for its extended performance. The factor may be evaluated by calculating the central frequency $\left(f_{C}\right)$ of the antenna as the geometric mean of upper $\left(f_{H}\right)$ and lower $\left(f_{L}\right)$ 


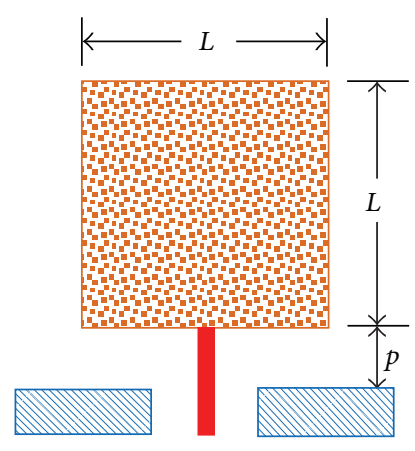

(a)

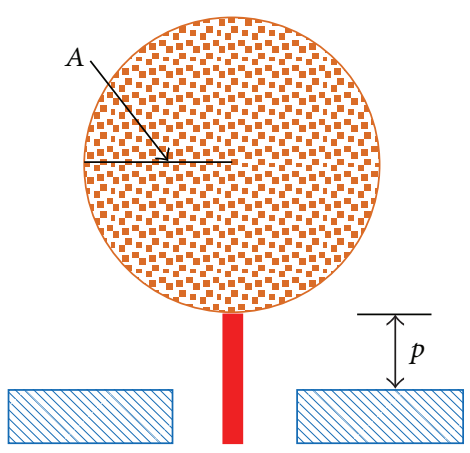

(b)

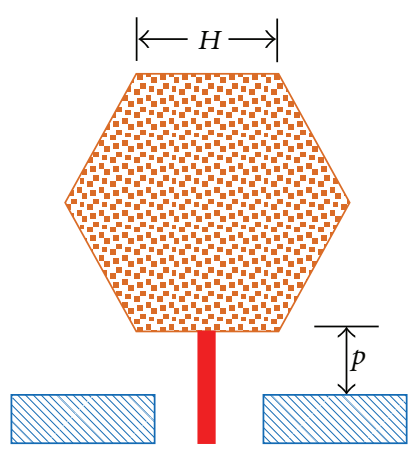

(c)

Figure 2: Geometrical shapes of PSMA, PCMA, and PHMA.

frequencies. The following relations will be used to calculate bandwidth and quality factor [13-17]:

$$
\begin{gathered}
f_{C}=\sqrt{f_{H} f_{L}} \mathrm{GHz}, \\
\mathrm{BW}=f_{H}-f_{L} \mathrm{GHz}, \\
\mathrm{Q}=\frac{f_{C}}{\mathrm{BW}}=\frac{\sqrt{f_{H} f_{L}}}{f_{H}-f_{L}} .
\end{gathered}
$$

As recommended by Federal Communication Commission in 2002 [2], the value of $f_{H}$ (upper band edge frequency) is set at $10.6 \mathrm{GHz}$.

By employing particle swarm optimization and using combine cost function for all three antennas, we achieve the joint optimized results.

\section{Particle Swarm Optimization}

Particle swarm optimization is the global heuristic algorithm developed in mid 1990s by Dr. Kennedy and Eberhart [18]. The algorithm was inspired by the "information sharing" capability within the groups of animals, such as birds' flocks, cattle's herds, to find the easiest and safest path towards food source [19]. The study was based on the behavior of birds' swarm movement. The purpose of the research was to investigate the social behavior and the collective intelligence of the birds' swarm.

Initially, during searching for food, the birds can either fly together in the form of a flock or they can move individually in different directions. While searching, there must be at least one bird that first smells the food. That is the one who has the best information about the location of the food or at least knows the direction where the food is located. The bird shares its "knowledge" to others, and then eventually all the birds reach at the source of food [20].

The PSO is used to find the optimistic solution of complex problems. In PSO algorithm, Kennedy and Eberhart compared the solution swarm with the bird's swarm. The development of solution swarm is compared with the movement of birds from one point to another. Also, the optimized solution in PSO is equivalent to the targeted food resource for birds' swarm.
The algorithm propagates through a randomly generated population known as particles. Each particle iteratively moves with a random velocity in the designed problem space towards the targeted optimum solution. The particle is moving towards the location which was best known to it and then moves iteratively towards the best fitness known by whole population [21].

There are three main steps of standard PSO [22].

Initialization. In this step, population (swarm) is assigned some random velocity $v_{k}^{i}$ and position $x_{k}^{i}$ to $i$ th particle at time instant $k$. A particle is a "point" in the designed space that moves iteratively. The particle changes its location (position) from one move (iteration) to another move depending on its update velocity.

This step allows particles to be randomly distributed in the entire designed space. Equations (9) generate the random position and velocity of a particle using upper and lower bounds $x_{\min }$ and $x_{\max }$,

$$
\begin{gathered}
\mathbf{x}_{0}^{i}=\mathbf{x}_{\min }+\operatorname{rand}\left(\mathbf{x}_{\max }-\mathbf{x}_{\min }\right), \\
\mathbf{v}_{0}^{i}=\frac{\text { position }}{\text { time }}=\frac{\mathbf{x}_{\min }+\operatorname{rand}\left(\mathbf{x}_{\max }-\mathbf{x}_{\min }\right)}{\Delta t} .
\end{gathered}
$$

Velocity Update. The next step calculates the update velocity at time instant $k+1$. The update is based on the fitness value of particle current position.

The fitness function value determines the particle having the best global value $p_{k}^{g}$ in the current population. It also determines the best position of each particle $p^{i}$. The update velocity at time instant $k+1$ is given in the following:

$$
\mathbf{v}_{k+1}^{i}=w \mathbf{v}_{k}^{i}+c_{1} \text { rand } \frac{\left(\mathbf{p}^{i}-\mathbf{x}_{k}^{i}\right)}{\Delta t}+c_{2} \operatorname{rand} \frac{\left(\mathbf{p}_{k}^{g}-\mathbf{x}_{k}^{i}\right)}{\Delta t}
$$

The right side of (10) is the summation of three important terms. These terms are the current particle motion, the memory influence of the particle, and the swarm influence. 


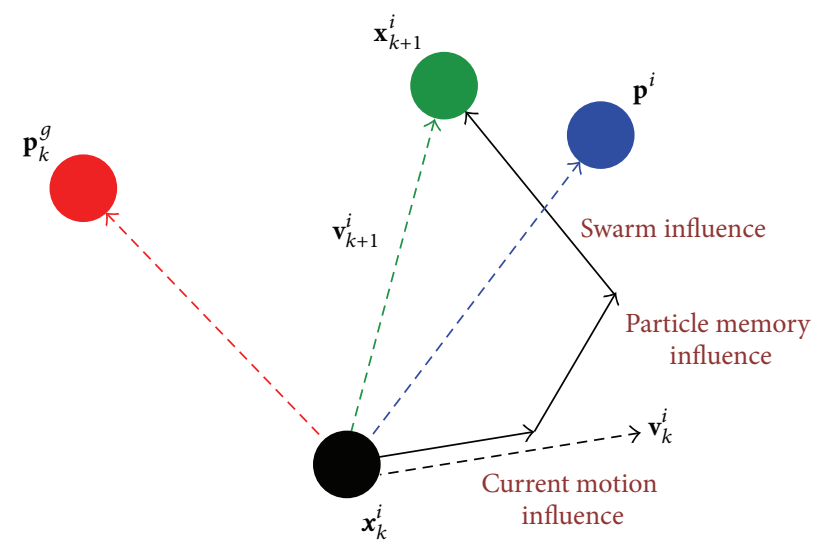

FIGURE 3: Illustration of velocity and position updates in PSO algorithm.

The constants $w, c_{1}$, and $c_{2}$ are the inertia factor, particle selfconfidence, and swarm confidence, respectively.

Position Update. The last step describes the update position of a particle at time instant $k+1$. This position update is given in the following:

$$
\mathbf{x}_{k+1}^{i}=\mathbf{x}_{k}^{i}+\mathbf{v}_{k+1}^{i} \Delta t .
$$

Figure 3 shows the illustration of velocity and position updates using particle swarm optimization.

\section{Development of Cost Function}

By employing the above optimization technique mentioned in Section 3, we are now going to develop the cost function for our problem. Generalized equations of error for these antennas, namely, frequency error $\left(\right.$ Error $\left._{f L}\right)$, bandwidth error $\left(\right.$ Error $\left._{\mathrm{BW}}\right)$, and quality factor error $\left(\right.$ Error $\left._{\mathrm{Q}}\right)$, are as follows:

$$
\begin{gathered}
\text { Error }_{f_{L}}=e_{1}=f_{L \text { desired }}-f_{L \text { calculated }} \mathrm{GHz}, \\
\text { Error }_{\mathrm{BW}}=e_{2}=\mathrm{BW}_{\text {desired }}-\mathrm{BW}_{\text {calculated }} \mathrm{GHz}, \\
\text { Error }_{\mathrm{Q}}=e_{3}=Q_{\text {desired }}-Q_{\text {calculated }} .
\end{gathered}
$$

Combining all the above error effects in one relation for one particular antenna, following mean square error (MSE) relations which are employed for all types of antennas under consideration:

$$
\begin{aligned}
\operatorname{MSE}_{\mathrm{PSMA}} & =\sum_{i=1}^{3} w_{i} e_{i(\mathrm{PSMA})}^{2}, \\
\mathrm{MSE}_{\mathrm{PCMA}} & =\sum_{i=1}^{3} w_{i} e_{i(\mathrm{PCMA})}^{2}, \\
\operatorname{MSE}_{\mathrm{PHMA}} & =\sum_{i=1}^{3} w_{i} e_{i \text { (PHMA) }}^{2} .
\end{aligned}
$$

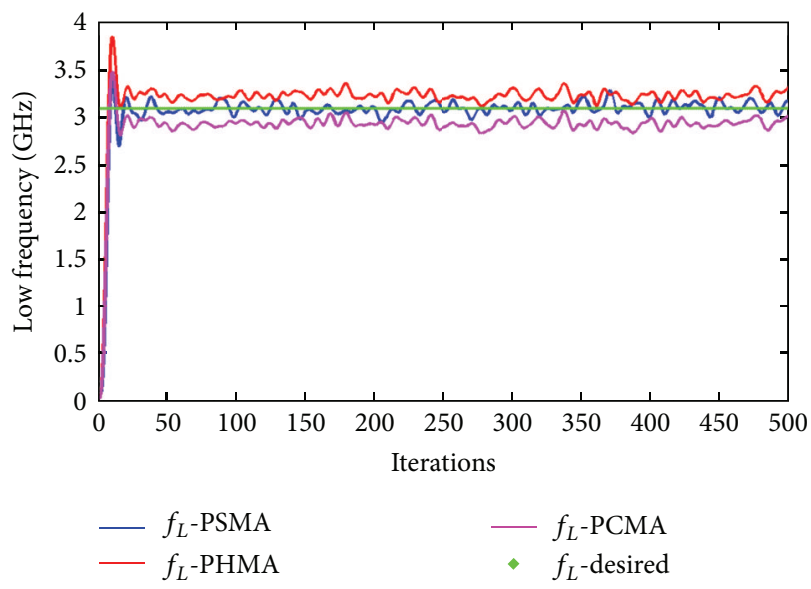

FIGURE 4: Optimized values of lower frequencies for PSMA, PCMA, and PHMA.

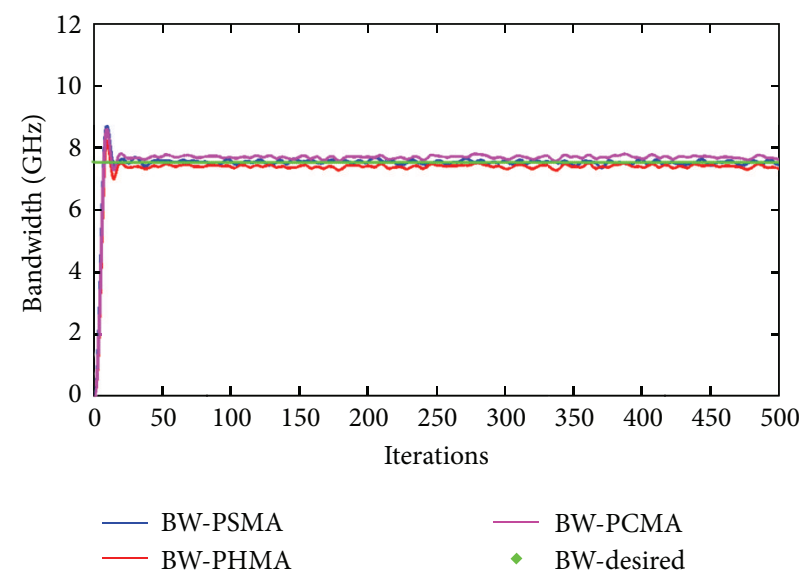

FIGURE 5: Optimized values of bandwidths for PSMA, PCMA, and PHMA.

Here $w_{i}$ is the random weight for all the errors. For simplicity, we have kept the total sum of all three weights equal to one. Whereas $e_{i}$ represents three different errors (separately for PSMA, PCMA, and PHMA) as described in the above equations (12)-(14).

The joint mean square error (cost function) for these antennas is given in the following:

$$
\text { Cost function }=\frac{\left(\mathrm{MSE}_{\mathrm{PSMA}}+\mathrm{MSE}_{\mathrm{PCMA}}+\mathrm{MSE}_{\mathrm{PHMA}}\right)}{3} .
$$

Using particle swarm optimization we have minimized this cost function to get optimized geometric dimensions of the three antennas at the same targeted lower band edge frequency, bandwidth, and quality factor.

The geometric dimensions for PSMA are the length (or width) of square monopole $L$ and the length of the feed line from ground surface $p$; the geometric dimensions for PCMA are the radius of the circular patch $A$ and the length of the feed line from ground surface $p$, whereas the geometric 


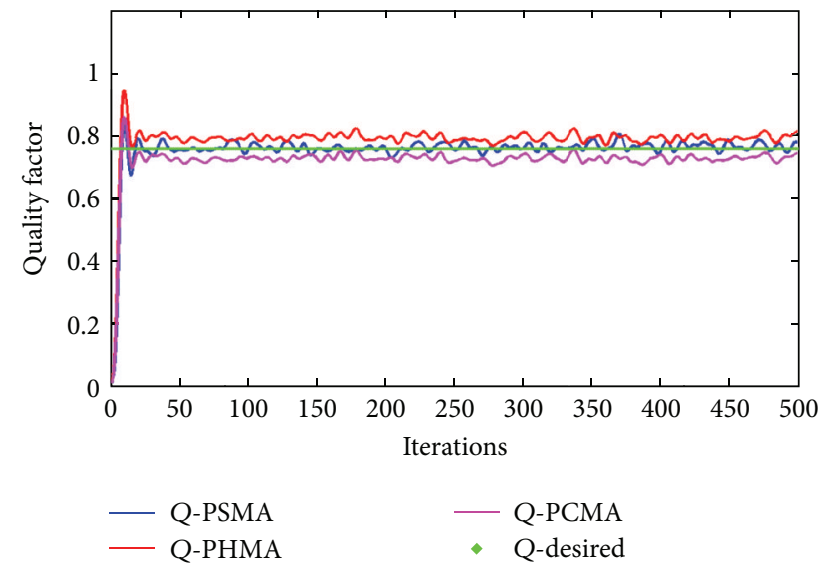

FIGURE 6: Optimized values of quality factors for PSMA, PCMA, and PHMA.

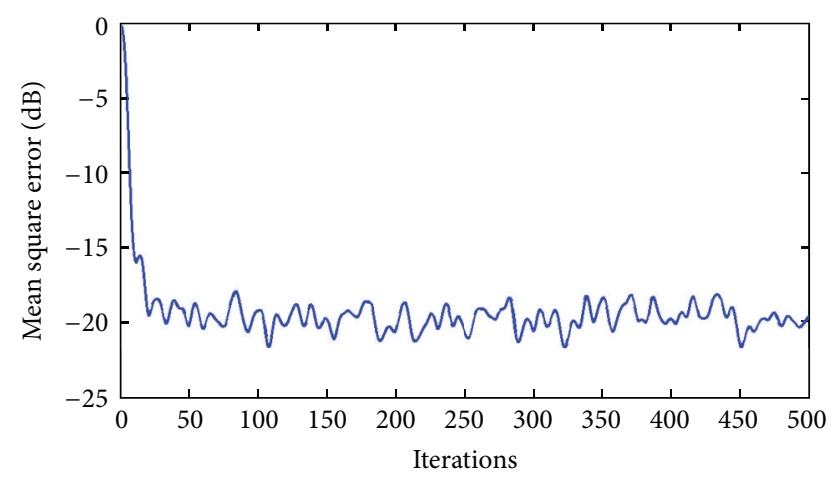

FIgURE 7: Optimized value of joint cost function of PSMA, PCMA, and PHMA.

dimensions for PHMA are the length of the sides of hexagon monopole $H$ and the length of the feed line from ground surface $p$.

Tables 1-3 show optimized geometric dimensions of PSMA, PCMA, and PHMA antennas, respectively. The tables show few desired values of lower band edge frequency, bandwidth, and quality factor within the frequency range of UWB (from $3.1 \mathrm{GHz}$ to $10.6 \mathrm{GHz}$ ).

Different sets of frequency, bandwidth, and quality factor have been recorded in Tables 1-3. Against each set, jointly optimized values of $L, A, H$, and $p$ have been achieved for PSMA, PCMA, and PHMA.

Though there are numerous readings which have been observed, however graphical optimization results are shown for one specific set only. Since as per FCC, $3.1 \mathrm{GHz}$ is allocated as lower frequency for UWB spectrum; all the graphical representations from Figures 4, 5, 6, and 7 are at this value. These figures show the iterative calculated values of lower band edge frequency $\left(f_{L}\right)$, bandwidth (BW), quality factor $(Q)$, and joint cost function for PSMA, PCMA, and PHMA with 500 iterations.

\section{Conclusion}

Three different shaped printed monopole antennas PSMA, PCMA, and PHMA have been jointly optimized for UWB applications. Particle swarm optimization has been successfully used as a heuristic approach to minimize the joint cost function and to calculate the optimized values of the geometric dimensions of all three antennas. The promising results provide a framework for the future in designing any shape of antenna for any range of frequency.

\section{References}

[1] "Ultra-wideband technology—enabling high speed wireless personal area networks," A White Paper by Intel 300982-002 US, 2005.

[2] "FCC report and order on ultra wideband technology," Tech. Rep. FCC 02-48, Federal Communications Commission, Washington, DC, USA, 2002.

[3] M. Hammoud, P. Poey, and F. Colombel, "Matching the input impedance of a broadband disc monopole," Electronics Letters, vol. 29, no. 4, pp. 406-407, 1993.

[4] G. Kumar and K. P. Ray, Broad Band Microstrip Antennas, Artech House, Boston, Mass, USA, 2003.

[5] N. P. Agrawall, G. Kumar, and K. P. Ray, "Wide-band planar monopole antennas," IEEE Transactions on Antennas and Propagation, vol. 46, no. 2, pp. 294-295, 1998.

[6] H. G. Schantz, "Planar elliptical element ultra-wideband dipole antennas," in Proceedings of the IEEE Antennas and Propagation Society International Symposium, vol. 3, pp. 44-47, June 2002.

[7] O. T. Altinoz and A. E. Yilmaz, "Calculation of optimized parameters of rectangular patch antenna using gravitational search algorithm," in Proceedings of International Symposium on Innovations in Intelligent Systems and Applications (INISTA '11), pp. 349-353, June 2011.

[8] S. Sharma and B. K. Kanaujia, "Optimization of resonant frequency of circular microstrip antenna with and without air gaps using bacterial foraging optimization technique," in Proceedings of International Conference on Computational Intelligence and Communication Networks (CICN '11), pp. 574-577, 2011.

[9] V. S. Chintakindi, S. S. Pattnaik, O. P. Bajpai, S. Devi, S. V. R. S. Gollapudi, and P. K. Pradyumna, "Parameters calculations of rectangular microstrip patch antenna using particle swarm optimization technique," in Proceedinga of IEEE Applied Electromagnetics Conference (AEMC '07), pp. 1-4, December 2007.

[10] W. C. Liu, "Design of a multiband CPW-fed Monopole antenna using a particle swarm optimization approach," IEEE Transactions on Antennas and Propagation, vol. 53, no. 10, pp. 32733279, 2005.

[11] M. T. Islam, N. Misran, T. C. Take, and M. Moniruzzaman, "Optimization of microstrip patch antenna using particle swarm optimization with curve fitting," in Proceedings of International Conference on Electrical Engineering and Informatics (ICEEI '09), vol. 2, pp. 711-714, August 2009.

[12] N. Jin and Y. Rahmat-Samii, "Parallel particle swarm optimization and finite-difference time-domain (PSO/FDTD) algorithm for multiband and wide-band patch antenna designs," IEEE Transactions on Antennas and Propagation, vol. 53, no. 11, pp. 3459-3468, 2005.

[13] K. P. Ray, "Design aspects of printed monopole antennas for ultra wide band applications," International Journal of Antennas and Propagation, vol. 2008, Article ID 713858, 8 pages, 2008. 
[14] N. Hecimovic and Z. Marincic, The Improvements of the Antenna Parameters in Ultra-Wideband Communications, Ericsson Nikola Tesla, Zagreb, Croatia, 2008.

[15] H. Schantz, The Art and Science of Ultra-Wideband Antennas, Artech House, London, UK, 2005.

[16] L. J. Chu, "Physical limitations of omni-directional antennas," Journal of Applied Physics, vol. 19, no. 12, pp. 1163-1175, 1948.

[17] J. S. McLean, "A re-examination of the fundamental limits on the radiation Q of electrically small antennas," IEEE Transactions on Antennas and Propagation, vol. 44, no. 5, pp. 672-676, 1996.

[18] J. Kennedy and R. Eberhart, "Particle swarm optimization," in Proceedings of the IEEE International Conference on Neural Networks, pp. 1942-1948, Perth, Australia, December 1995.

[19] J. Kennedy and R. Eberhart, Swarm Intelligence, Academic Press, San Diego, Calif, USA, 1st edition, 2001.

[20] Q. Bai, "Analysis of particle swarm optimization algorithm," Computer and Information Science, vol. 3, no. 1, pp. 180-184, 2010.

[21] I. C. Trelea, “The particle swarm optimization algorithm: convergence analysis and parameter selection," Information Processing Letters, vol. 85, no. 6, pp. 317-325, 2003.

[22] R. Hassan, B. Cohanim, O. de Weck, and G. Venter, "A comparison of particle swarm optimization and the genetic algorithm," American Institute of Aeronautics and Astronautics, 2004. 

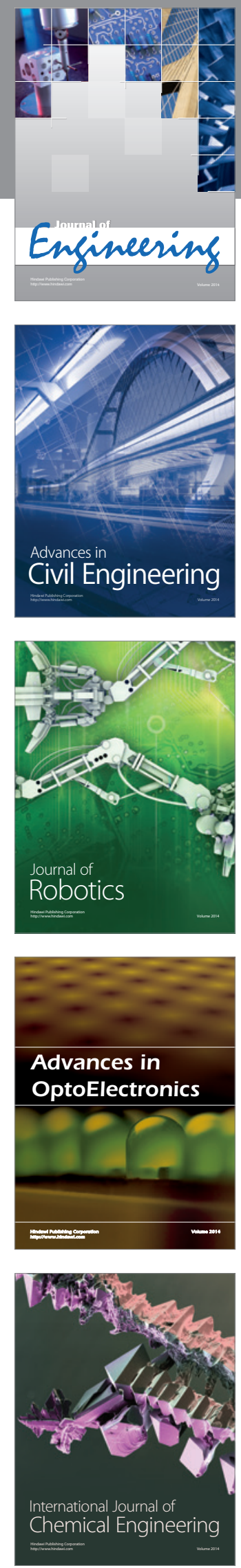

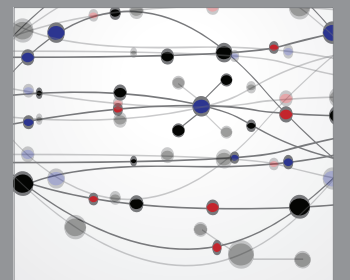

The Scientific World Journal
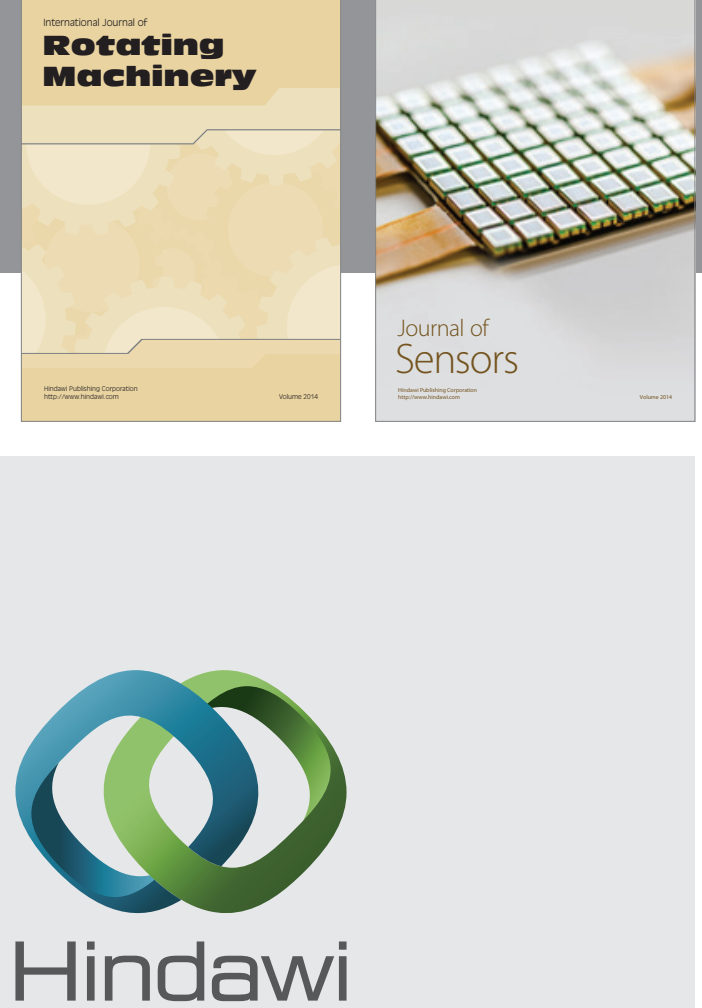

Submit your manuscripts at http://www.hindawi.com
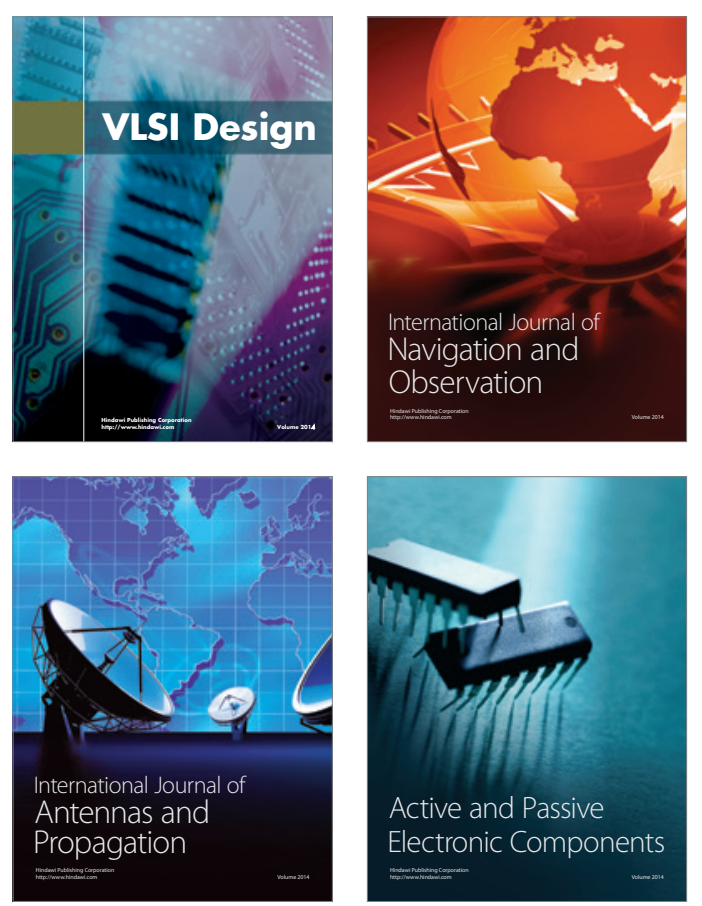
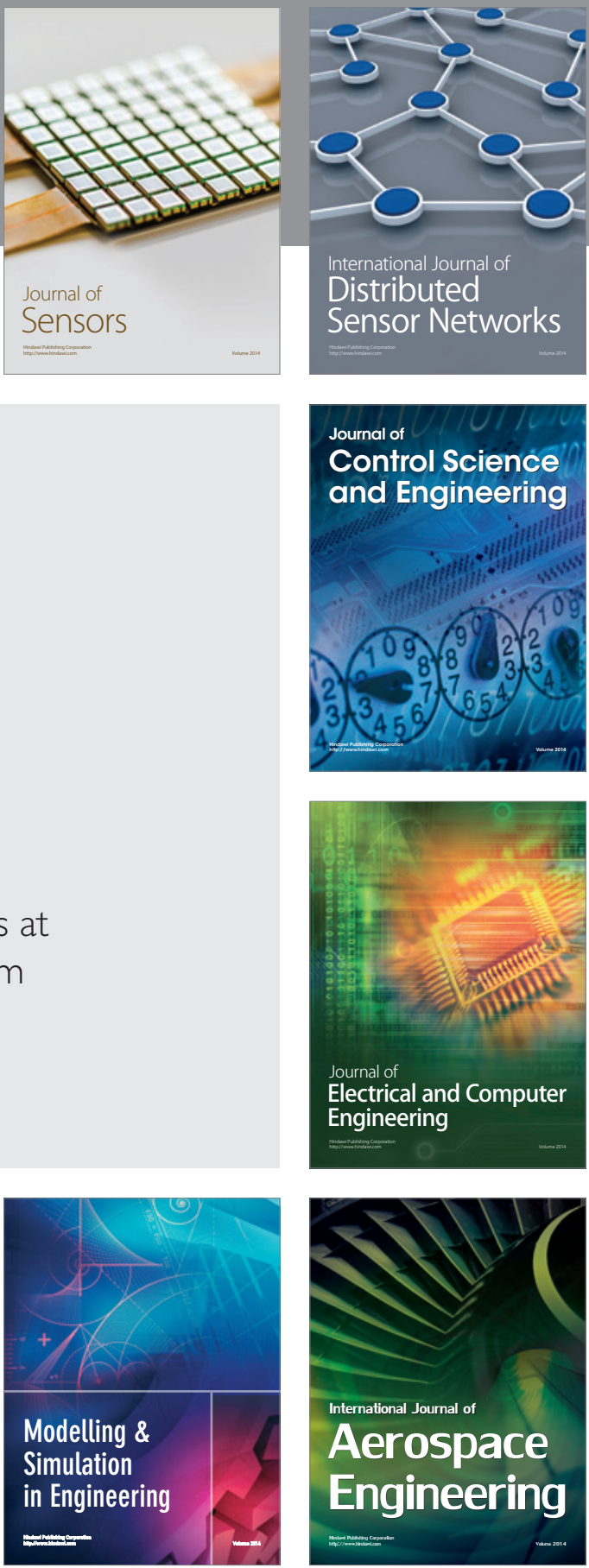

Journal of

Control Science

and Engineering
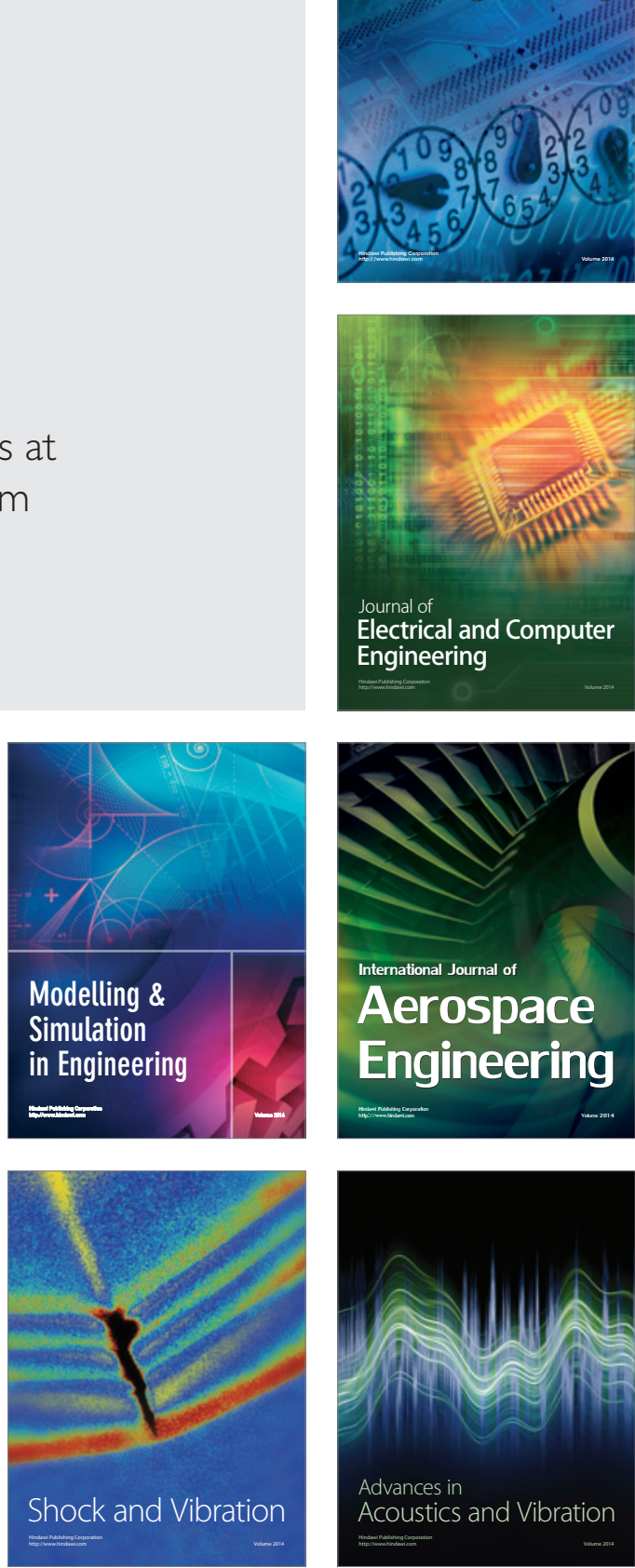